\title{
HUMAN BODY DETECTION AND SAFETY CARE SYSTEM FOR A FLYING ROBOT
}

\author{
Bahram Lavi Sefidgari \\ Department of Computer Engineering, \\ Eastern Mediterranean University, Cyprus \\ bahram. lavi@emu. edu.tr
}

\begin{abstract}
Image-processing is one the challenging issue in robotic as well as electrical engineering research contexts. This study proposes a system for extract and tracking objects by a quadcopter's flying robot and how to extract the human body. It is observed in image taken from real-time camera that is embedded bottom of the quadcopter, there is a variance in human behaviour being tracked or recorded such as position and, size, of the human. In the regard, the paper tries to investigate an image-processing method for tracking humans' body, concurrently. For this process, an extraction method, which defines features to distinguish a human body, is proposed. The proposed method creates a virtual shape of bodies for recognizing the body of humans, also, generate an extractor according to its edge information. This method shows better performance in term of precision as well as speed experimentally.
\end{abstract}

\section{KEYWORDS}

Image processing, Human detection, Tracking, Quadcopter, Flying robot

\section{INTRODUCTION}

Quadcopter, also known as quadrotor, is a helicopter with four rotors. The rotors are directed upwards and they are placed in a square configuration with equal distance from the center of mass of the quadcopter. The quadcopter is controlled by adjusting the angular velocities of the rotors which are spun by electric motors. Quadcopter is a usual design for small unmanned aerial vehicles (UAV) because of the simple structure. This robot has been used in supervision, search and rescue, construction inspections and several other applications $[1,2,3]$.

Regarding their complicate structure, the quadcopter is these days taken into consideration by many of the robotics researches and its complication causes special abilities which can be used in broad range of usages [4,5].Quadcopter unmanned aerial vehicles (UAV) are used for supervision and reconnaissance by military and law enforcement agencies, as well as search and rescue missions in urban environments, which is a small UAV that can quietly hover in place and use camera to tracking people on the ground.

Recently, many studies are being done for providing a proper detecting method which can track the persons in different situations with high efficiency. Although, several studies have been done for detect and tracking human either from a fixed camera station or from a camera which has 
motion, there is limited contributions for real-time human track and/ or detect from camera which is installed on a real quadcopter. The main modules which are vital for image processing using a quadcopter are; wireless virtual interfaces, low bandwidth video compression [2]. Having had a constant altitude is also important for capturing high quality image [3].Quadcopter which was shown in Figure 1 is an outdoor testbed for testing.

The aim of this research is developing a real-time system for detect as well as tracking of the humans by a quadcopter. In this regards we applied edge detection approach. The system is under implementation experimentally. The main object of this work was employing quadcopter as a safety and security robot at the wide range areas.

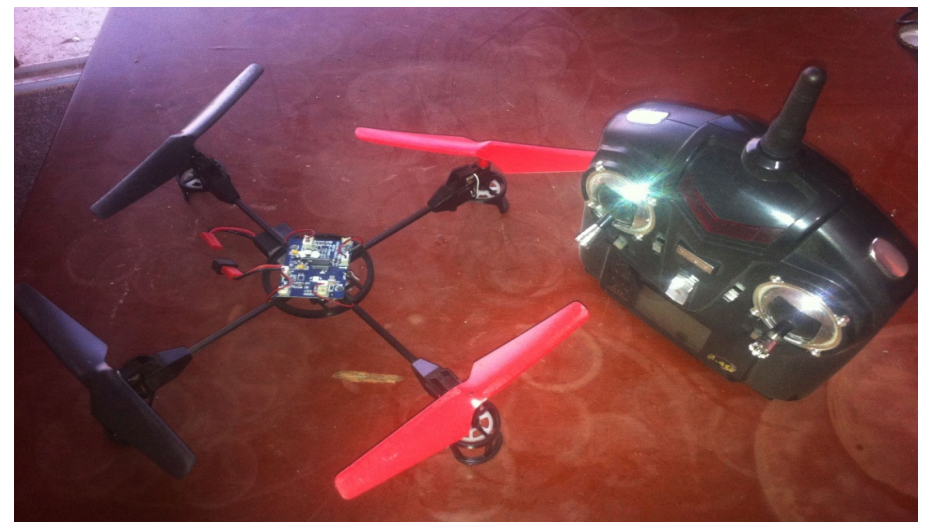

Figure 1. Experimental mini quadcopter

\section{RELATED WORKS}

\subsection{Quadcopter dynamic model}

Quadcopter flying robot is controlled by changeable the angular speed of each motor. It has for rotors arranged in cross shape. The front and back rotors are rotating counter clockwise direction and the left and right rotors are rotating clockwise side [6] which was shown in Figure 2 which is whole rotating structure of rotors in a quadcopter.

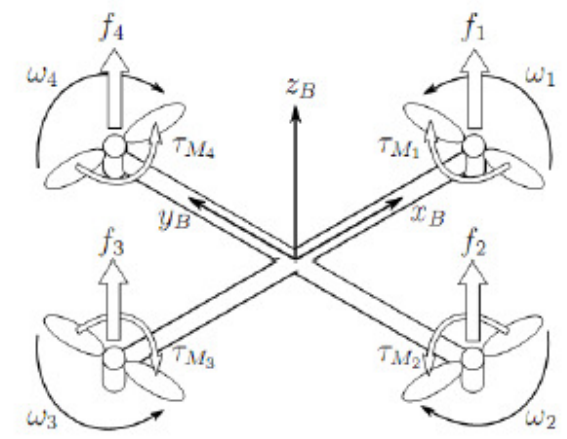

Figure 2. Whole structure of rotation of rotors in quadcopter

The flying robot considered in this work is classified as a mini flying robot with limited weight to less than $1 \mathrm{~kg}$. Integration of a homemade flying robot with altitude controlling system [3] and a small camera are required in order to have an adequate embedded image-processing platform. The prototype built to test the image processing feedback with detail is shown in Figure 3 


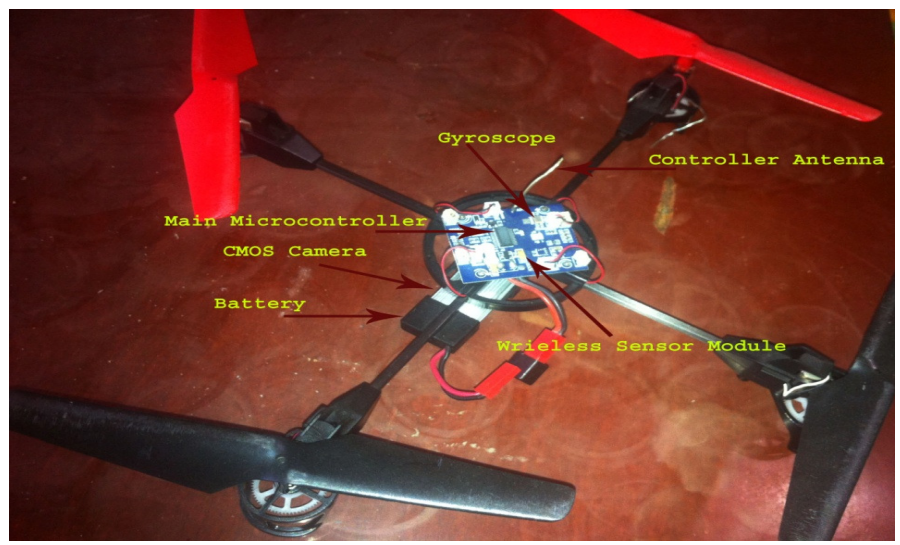

Figure 3. Structure of home-made quadcopters

The control of quadcopter is applied PID controller, that the coefficients of which was improved by genetic algorithm [3], which is implemented on AVR microcontroller. The performance of the overall system is valuated in real-time experiments. Also we embedded a small CMOS camera module with photographic array $320 * 240$ (see Figure 4) for implementing image processing, and wireless sensor module (see Figure 5) to have transferring data between quadcopter and Server.

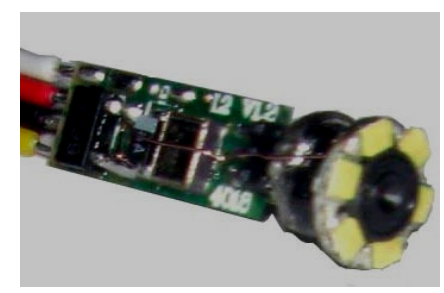

Figure 4. CMOS camera module

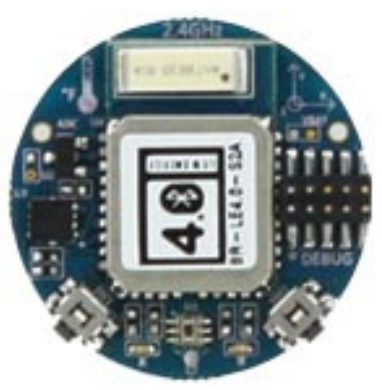

Figure 5. Wireless sensor module

\subsection{Image-processing in quadcopter}

Human detection systems consist of body extraction and classification. To the best of our knowledge, most of the human detection algorithms are different in feature extraction while they usually use classical tracking such as support vector machines [7-11] and Adaboost [12-14]. Also body extraction system by flying robot, such a quadcopters, is still a hot topic and open subject for human detection. 
Although there are many algorithms for image processing are developed based on human detection, such as; HOG and LBP [15], Haar wavelets and EOH [16], region covariance matrix [17-18], partial least squares [19], edge detection[1], stereo vision [20-23], monocular vision [22],[25], sonar and vision [28], laser and vision [24], and thermal vision [26-29], but we couldn't find any work about human body detection by microcontrollers for quadcopter, and at this paper the edge detection algorithm is employed for detecting human body by quadcopter.

Detection human in real-life environment is a challenging subject, mostly because of some variables are changeable such as condition, background, and human body. In fact, human body is a complex object with several degrees of freedom, whose body formation can change greatly onto a 2D image. Thus, the problem of a human body using standard CMOS camera that embedded in bottom of quadcopter.

\section{HUMAN DETECTION METHODS ON QUADCOPTER}

\subsection{System Overview}

A typical object tracking system extract any movements, recognizes an object from the movement and applies geometric algorithms to check whether the object is a human. The extraction of human body could be used instead for fixing altitude of quadcopter but it requires normalization. A histogram geometric algorithm performs precise extraction, and at its final stage, a geometric algorithm is applied to detect human body within small object area and send a message to server include of detecting some humans. Figure 5 presents the diagram of the proposed system.

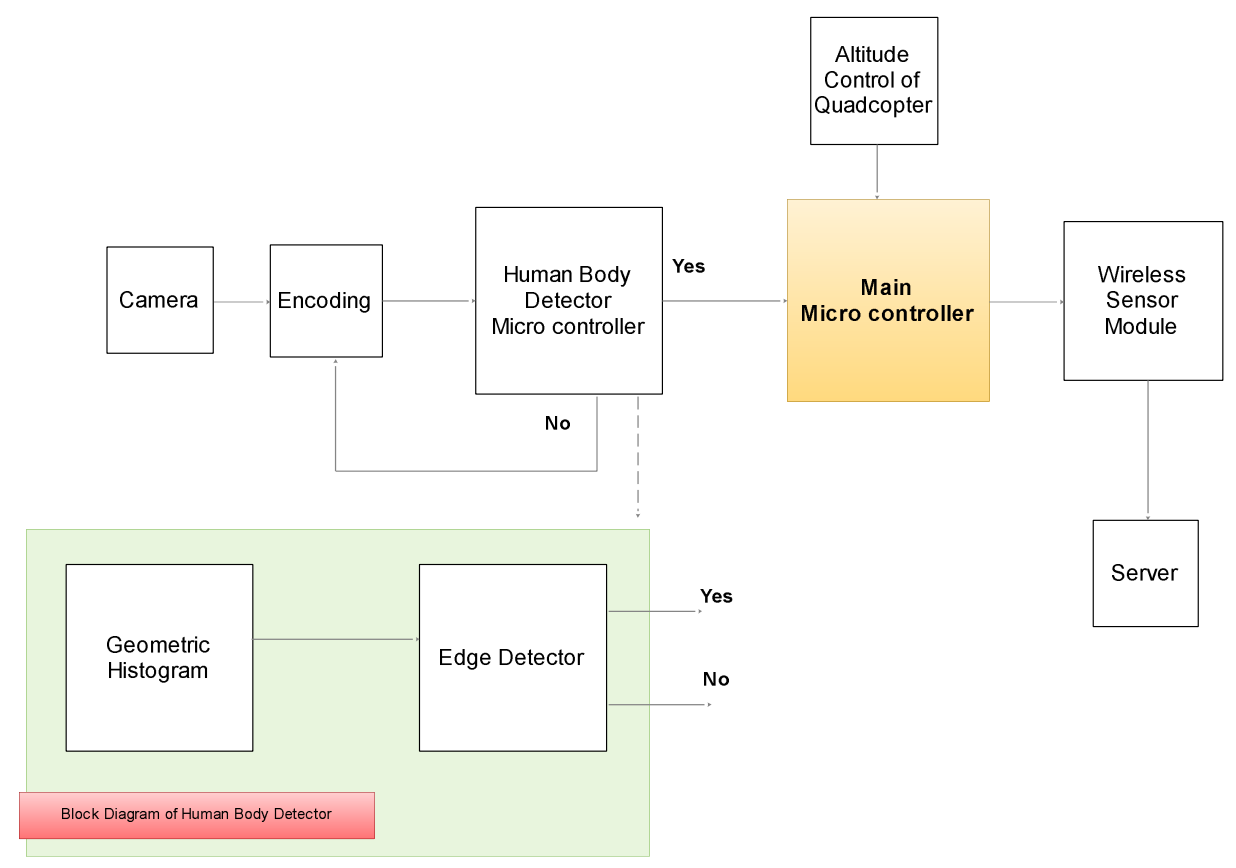

Figure 6. Block diagram of human detection in quadcopter

The main module can be grouped in two categories, human body detection and decision making with the main micro controller for making order to fix altitude of quadcopter and send signals to the wireless sensor module. 
First, camera take image and using with encoder, to convert data in binary format, human body detector micro controller are used in order to filling dataset. Then the information contained in these sets in integrated and analyzed using edge detector. This module performs the key task of the detection of human body. Among other tasks, control for fixing altitude of quadcopter is provided. Table 1 list the sub modules and methods used in each module. The main modules are detailed in sections below.

Figure 7 presents an example of the output of some modules. It is important to note human body detector module work on gray-scale images.

Table 1. List of modules and methods

\begin{tabular}{|l|l|l|l|}
\hline \multicolumn{1}{|c|}{ Module name } & \multicolumn{1}{|c|}{ Sub module } & \multicolumn{1}{c|}{ Output } & \multicolumn{1}{c|}{ Method } \\
\hline Encoding & - & $\begin{array}{l}\text { Binary data of } \\
\text { images }\end{array}$ & Dynamic programming \\
\hline $\begin{array}{l}\text { Human body } \\
\text { detector }\end{array}$ & Geometric histogram & Human Object & Pattern machine \\
Edgetector & $\begin{array}{l}\text { Tracking human } \\
\text { bodies }\end{array}$ & $\begin{array}{l}\text { Medina filter and } \\
\text { morphology operation } \\
\text { [1] }\end{array}$ \\
\hline $\begin{array}{l}\text { Main micro } \\
\text { controller }\end{array}$ & - & $\begin{array}{l}- \text { Signal for fixing } \\
\text { altitude of } \\
\text { quadcopter } \\
\text { Signal for wireless } \\
\text { sensor module }\end{array}$ & Dynamic programming \\
\hline $\begin{array}{l}\text { Altitude control of } \\
\text { quadcopter }\end{array}$ & - & $\begin{array}{l}\text { Feedback signal for } \\
\text { ever signal has been } \\
\text { received from main } \\
\text { microcontroller }\end{array}$ & $\begin{array}{l}\text { Neural fuzzy } \\
\text { Genetic algorithm [3] }\end{array}$ \\
\hline $\begin{array}{l}\text { Wireless sensor } \\
\text { module }\end{array}$ & - & $\begin{array}{l}\text { Send signal } \\
\text { including detecting } \\
\text { persons }\end{array}$ & Dynamic programming \\
\hline
\end{tabular}

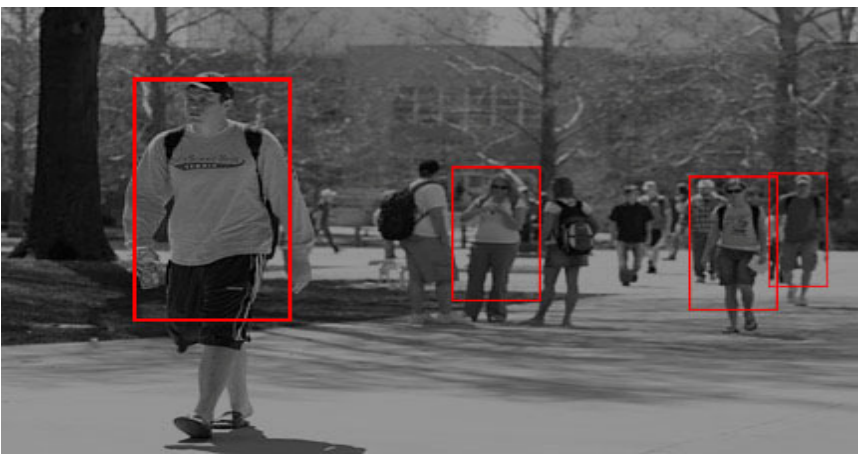

\subsection{Encoding}

Figure 7. Example of human body detection via edge detection method

The encoding module converts an image, which has been received from camera, to binary image format. We convert this job by using dynamic programming in the modules. A binary image is a 
digital image that has only two possible values for each pixel. Numerically, the values are often 0 for black and either 1 or 255 for white.

\subsection{Human body detector}

The geometric histogram is a based on a histogram of human body models is created in the horizontal and the vertical directions. To implement this, geometrical objects are transformed to a simple binary image in order to diagnose human body objects via pattern machine [1]. The geometrical objects are used for human body detection.

Histograms are collected via the human body objects and the similarity of histograms is evaluated. The similarity is supplied using the Euclidian distance. To distinguish the human body object from other objects, accumulated histograms are calculated as:

$$
\operatorname{Similarity}\left(\operatorname{select}\left(H_{P 1} \approx H_{P 3}\right)\right.
$$

The proposed of the algorithm to detect human body is provided between binary image and updated binary image. Given that stored dataset are composed of many geometric histograms. For each histogram is taken to be a vector then degree of match between model feature $\mathrm{Mj}$ and image feature I is accumulated as:

$$
D_{J}=\sum_{i=1}^{n} \sqrt{I_{i} \cdot M_{j i}}
$$

This is performed for each pixel of binary image and updated binary image to achieve degree of vectors.

When geometric histogram is done for the binary image, media filter and morphology operation should be implemented to detecting edges in the binary image.

The edge detector is to detect the area in binary image which a person stands and the body of that person, the system extracts body edge shapes to track the object. For the detection, median filter and morphology operation are applied from gray-scale differential images to remove noise while detecting the objects.

The median filter is an effective method that can overturn isolated noise without blurring sharp edge. Specially, the median filter replaces a pixel by the median of all pixels in the neighbourhood. This method is defined by:

$$
y[m, n]=\operatorname{meddian}\{x[i, j],(i, j) \in w\}
$$

Where w represents a neighbourhood centered on location $(\mathrm{m}, \mathrm{n})$ in the binary image.

The morphology operation converts a binary image into an image where every foreground pixel has a value corresponding to the minimum distance from the background. The algorithm is applied the Euclidean distance metric to transform the image. Firstly, for 2D Images, the algorithm determines the image resolution in $\mathrm{X}$ and $\mathrm{Y}$ directions. Then it is identified all edges pixels. And finally, it is created a distance metric, where each pixel of the metric is related with a matching area of the binary image. Each pixel is assigned a calculated distance value matching to the Euclidean distance a center of that pixel and the nearest point of the updated binary image. For tow $2 \mathrm{D}$ point $\mathrm{P}(\mathrm{x} 1, \mathrm{y} 1)$ and $\mathrm{Q}(\mathrm{x} 2, \mathrm{y} 2)$ the classical Euclidian distance is defined by: 


$$
\sqrt{\left(x_{2}-x_{1}\right)^{2}+\left(y_{2}-y_{1}\right)^{2}}
$$

The background distance operation when calculates the Euclidian distance take into account the image resolutions in $\mathrm{X}$ and $\mathrm{Y}$ dimensions. So that it is defined by:

$$
\sqrt{\left(x_{2}-x_{1}\right)^{2} \times x_{\text {resolution }}^{2}+\left(y_{2}-y_{1}\right)^{2} \times y_{\text {resolution }}^{2}}
$$

\subsection{Main micro controller}

Output data from quadcopter is sent through wireless sensor module. The data is outputted using that and data is transmitted to transmitter at server. Based on that data controller send command signal and number of detected human body to server and server responses are sensed using receiver. Figure 6 shows an application which is implemented on the $\mathrm{C \#}$ programming language to ensure achievement of command signals. Figure 8 presents a form of application which has been implemented.

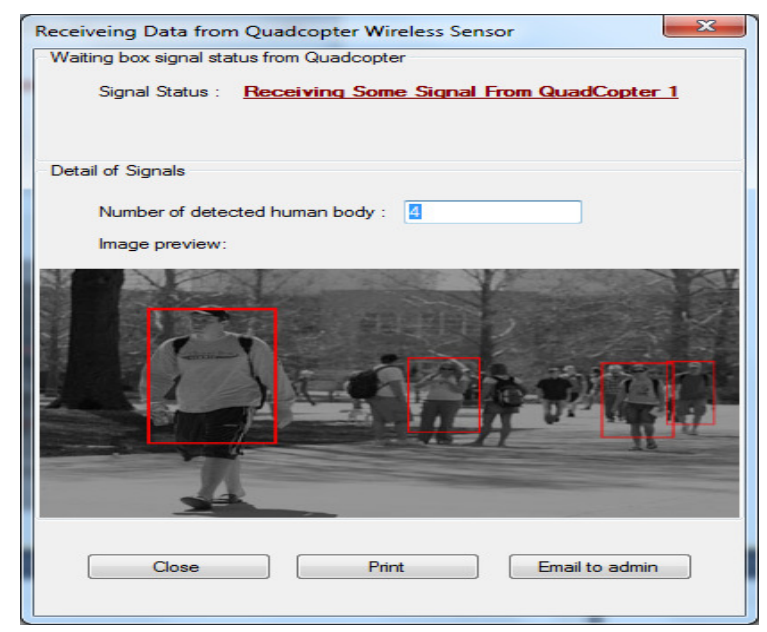

Figure 8. Application form of receiver on server when get some signal from quadcopter

Also, using Neuro PID and genetic algorithms which is applied in the micro controller [3], for controlling altitude of quadcopters are used. This is impossible to have a clear image in movement of quadcopters.

\section{Conclusions}

The development of flying robots is a challenging task. One of the most basic problems is how to enable to detect humans by flying robot. In this paper, an edge detection-based human body have been developed which is applied in a homemade flying robot. An edge detection method is performed to detect human body objects, and to process them further in order to send some command signal to server which included human numbers and one preview image of result via quadcopter. The images taken by the developed system shows acceptable outcomes. Also, the altitude control system which is used in this experiment largely improves the quality of the images, however, darkness and fog is a challenging issue. The developed system is a good starting point for expending image processing in flying robots aiming as a safety and security system. 


\section{REFERENCES}

[1] Lee, K. D., Nam, M. Y., Chung, K. Y., Lee, Y. H., \& Kang, U. G. (2013), "Context and profile based cascade classifier for efficient people detection and safety care system", Multimedia Tools and Applications, 1-18.

[2] Wren, C. R., Azarbayejani, A., Darrell, T., \& Pentland, A. P. (1997). Pfinder: Real-time tracking of the human body. Pattern Analysis and Machine Intelligence, IEEE Transactions on, 19(7), 780-785.

[3] Fatan,M.,Lavi,B.,Vatankhah,A.," An Adaptive Neuro PID for Controlling the Altitudeof Quadcopter Robot",18th international conference on methods and models in automation and robotics, August 2013

[4] Teppo Luukkonen, "Modelling and control of quadcopter," Independent research project in applied mathematics, Espoo, August 22, 2011.

[5] H. Huang, G. M. Hoffmann, S. L. Waslander, and C. J. Tomlin, "Aerodynamics and control of autonomous quadrotor helicopters in aggressive maneuvering," IEEE International Conference on Robotics and Automation, pp. 3277-3282, May 2009.

[6] González, I., Salazar, S., Torres, J., Lozano, R., \& Romero, H. (2013). Real-Time Attitude Stabilization of a Mini-UAV Quad-rotor Using Motor Speed Feedback. Journal of Intelligent \& Robotic Systems, 70(1-4), 93-106.

[7] X. Li, S. Lin, S. Yan, and D. Xu, "Discriminant locally linear embedding with high-order tensor data,” IEEE Trans. Syst., Man, Cybern. B, Cybern.,vol. 38, no. 2, pp. 342-352, Apr. 2008.

[8] Q. Zhu, M. Yeh, K. Cheng, and S. Avidan, "Fast human detection using a cascade of histograms of oriented gradients," in Proc. IEEE Conf. Comput. Vis. Pattern Recog., 2006, pp. 1491-1498.

[9] C. Burges, "A tutorial on support vectormachines for pattern recognition," Data Mining Knowl. Discov., vol. 2, no. 2, pp. 121-167, Jun. 1998.

[10] S. Maji, A. Berg, and J. Malik, "Classification using intersection kernel support vector machines is efficient," in Proc. IEEE Conf. Comput. Vis.Pattern Recog., 2008, pp. 1-8.

[11] Pang, Y., Yan, H., Yuan, Y., \& Wang, K. (2012). Robust coHOG feature extraction in humancentered image/video management system. Systems, Man, and Cybernetics, Part B: Cybernetics, IEEE Transactions on, 42(2), 458-468.

[12] Y. Chen and C. Chen, "Fast human detection using a novel boosted cascading structure with meta stages," IEEE Trans. Image Process., vol. 17, no. 8, pp. 1452-1464, Aug. 2008.

[13] M. Jones and P. Viola, "Fast multi-view face detection," Mitsubishi Elect. Res. Labs., Cambridge, MA, Tech. Rep. TR2003-096, 2003.

[14] P. Viola and M. Jones, "Detecting pedestrians using patterns of motion and appearance," in Proc. IEEE Int. Conf. Comput. Vis., 2003, vol. 2,pp. 734-741.

[15] X. Wang, T. Han, and S. Yan, "An HOG-LBP human detector with partial occlusion handling," in Proc. IEEE Int. Conf. Comput. Vis., 2009,pp. 32-39.

[16] D. Geronimo, A. Sappa, A. Lopez and D. Ponsa, "Adaptive image sampling and windows classification for on-board pedestrian detection,"in Proc. 5th Int. Conf. Comput. Vis. Syst., 2007.

[17] Y. Pang, Y. Yuan, and X. Li, "Gabor-based region covariance matrices for face recognition," IEEE Trans. Circuits Syst. Video Technol., vol. 18,no. 7, pp. 989-993, Jul. 2008.

[18] O. Tuzel, F. Porikli, and P. Meer, "Pedestrian detection via classification on Riemannian manifolds," IEEE Trans. Pattern Anal. Mach. Intell.,vol. 30, no. 10, pp. 1713-1727, Oct. 2008.

[19] R. Lienhart and J. Maydt, "An extended set of Haar-like features for rapid object detection," in Proc. IEEE Int. Conf. Image Process., 2002, vol. 1,pp. 900-903.

[20] Satake, J., Miura, J.: Robust Stereo-based Person Detectingand Tracking for a Person Following Robot. In:Proc. ICRA 2009 workshop on person detection and tracking. Kobe, Japan (2009)

[21] Wilhelm, T., Böhme, H.-J., Gross, H.-M.: A multimodal system for tracking and analyzing faces on a mobile robot. Robot. Auton. Syst. 48(1), 31-40, (2004),European Conference on Mobile Robots (ECMR '03)

[22] Medionia, G., R.J. Françoisa A., Siddiquia, M., Kima,K., Yoonb, H.: Robust real-time vision for a personal service robot. Comput. Vis. Image Understanding 108(1-2), 196-203, Special Issue on Vision for Human-Computer Interaction, October-November 2007

[23] Li, L., Koh, Y.T., Ge, S.S., Huang, W.: Stereo-based human detection for mobile service robots. Control, Automation, Robotics and Vision Conference, 2004, ICARCV, vol. 1, pp. 74-79 (2004)

[24] Bellotto, N., Hu, H.: Multisensor-based human detection and tracking for mobile service robots systems, man, and cybernetics, Part B: cybernetics. IEEE Trans.39(1), 167-181 (2009) 
[25] Böhme, H.J., Wilhelma, T., Keya, J., Schauera, C., Schrötera, C.,Großa,H-M., Hempelb, T.:An approach to multi-modal human-machine interaction for intelligentservice robots. Robot. Auton. Syst. 44(1), 83-96(2003)

[26] Meis, U., Oberlander, M., Ritter, W.: Reinforcing the reliability of pedestrian detection in far-infrared sensing. 2004 IEEE Intelligent Vehicles Symposium,pp. 779-783, 14-17 June 2004

[27] Mudaly, S.S.: Novel computer-based infrared pedestrian data-acquisition system. Electron. Lett. 15(13), 371-372 (1979)

[28] Nanda, H., Davis, L.: Probabilistic template based pedestrian detection in infrared videos. IEEE Intell. Veh. Symposium 1, 15-20 (2002)

[29] Bertozzi, M., Broggi, A., Fascioli, A., Graf, T., Meinecke, M.-M.: Pedestrian detection for driver assistance using multiresolution infrared vision. IEEE Trans. Veh. Technol. 53(6), 1666-1678 (2004)

\section{Authors}

Bahram Lavi Sefidgari received a B.S degree from Islamic Azad University of Tabriz, Iran. Currently, He is post graduate student computer engineering in Eastern Mediterranean University (EMU) and work in computer center of university. His research interests include Artificial Intelligent in robotics.

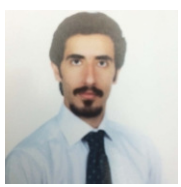

